\title{
Higher spins in hyperspace
}

\author{
loannis Florakis, ${ }^{a}$ Dmitri Sorokin ${ }^{b}$ and Mirian Tsulaia ${ }^{c}$ \\ ${ }^{a}$ Max-Planck-Institut für Physik, Werner-Heisenberg-Institut, \\ Föhringer Ring 6, 80805 München, Germany \\ ${ }^{b}$ INFN, Sezione di Padova, \\ via F. Marzolo 8, 35131 Padova, Italia \\ ${ }^{c}$ Faculty of Education Science Technology and Mathematics, University of Canberra, \\ University Dr, Bruce ACT 2617, Australia \\ E-mail: florakis@mppmu.mpg.de, dmitri.sorokin@pd.infn.it, \\ mirian.tsulaia@canberra.edu.au
}

ABSTRACT: We consider the $\operatorname{Sp}(2 n)$ invariant formulation of higher spin fields on flat and curved backgrounds of constant curvature. In this formulation an infinite number of higher spin fields are packed into single scalar and spinor master fields (hyperfields) propagating on extended spaces, to be called hyperspaces, parametrized by tensorial coordinates. We show that the free field equations on flat and AdS-like hyperspaces are related to each other by a generalized conformal transformation of the scalar and spinor master fields. We compute the four-point functions on a flat hyperspace for both scalar and spinor master fields, thus extending the two- and three-point function results of hep-th/0312244. Then using the generalized conformal transformation we derive two-, three- and four-point functions on AdS-like hyperspace from the corresponding correlators on the flat hyperspace.

KEywords: Conformal and W Symmetry, Conformal Field Models in String Theory, Field Theories in Higher Dimensions

ARXIV EPRINT: 1401.1645 


\section{Contents}

1 Introduction 1

2 Scalar and spinor field theory in flat hyperspace 4

3 Scalar and spinor field theory on the group manifold $\operatorname{Sp}(n) \quad 7$

4 GL-flatness of $\operatorname{Sp}(n)$ group manifolds and the relation between the field equations in flat and $\operatorname{Sp}(n)$ hyperspaces

$\begin{array}{lll}4.1 & \operatorname{Sp}(2 n) \text { transformations of the fields on } \operatorname{Sp}(n) & 11\end{array}$

$5 \mathrm{AdS}_{4}$ metric $\quad 12$

6 Correlation functions on $\operatorname{Sp}(n)$ group manifold 13

$\begin{array}{lll}6.1 & \text { Two-point functions } & 14\end{array}$

$\begin{array}{lll}6.2 & \text { Three-point functions } & 15\end{array}$

$\begin{array}{lll}6.3 & \text { Four-point functions } & 16\end{array}$

$\begin{array}{llr}7 & \text { Conclusion } & 18\end{array}$

A Proof of the GL( $n)$ flatness, some technical details and useful identities 20

\section{Introduction}

Various formulations of one and the same theory may prove useful for revealing and/or making manifest its different properties and features. This is certainly the case for higher spin gauge theory, for which various different descriptions have been proposed. Historically, the first approach was a metric-like formulation put forward by Fronsdal [1] and the second one was the frame-like approach $[2,3]$ which proved to be most efficient for constructing non-linear higher spin field theories with the use of unfolding techniques $[4-6]{ }^{1}$ Since consistent interactions require an infinite number of fields with spin ranging from zero to infinity, in this approach, massless higher spin gauge fields are encoded into a generalized (one-form) spin connection and a scalar (zero-form) field which, in the case of four spacetime dimensions $x^{m}(m=0,1,2,3)$, take the following form

$$
\begin{aligned}
& \omega(x, y, \bar{y})=\sum_{i, j=0}^{\infty} d x^{m} \omega_{m}^{\alpha_{1} \ldots \alpha_{i}, \dot{\beta}_{1} \ldots \dot{\beta}_{j}}(x) y_{\alpha_{1}} \ldots y_{\alpha_{i}} \bar{y}_{\dot{\beta}_{1}} \ldots \bar{y}_{\dot{\beta}_{j}}, \\
& C(x, y, \bar{y})=\sum_{i, j=0}^{\infty} C^{\alpha_{1} \ldots \alpha_{i}, \dot{\beta}_{1} \ldots \dot{\beta}_{j}}(x) y_{\alpha_{1}} \ldots y_{\alpha_{i}} \bar{y}_{\dot{\beta}_{1}} \ldots \bar{y}_{\dot{\beta}_{j}},
\end{aligned}
$$

\footnotetext{
${ }^{1}$ See reviews [7-14] and references therein for details on the features and different formulations of higher spin theory.
} 
where $y_{\alpha}$ and $\bar{y}_{\dot{\beta}}(\alpha, \dot{\beta}=1,2)$ are twistor-like Weyl-spinor variables, which are used to incorporate into a compact form an infinite number of physical higher spin gauge fields and their field strengths with spins $s$ growing from zero to infinity, as well as an infinite number of auxiliary fields. The variables $y_{\alpha}$ and $\bar{y}_{\dot{\beta}}$ can be regarded as coordinates that extend the conventional space-time with additional 'twistor-like' directions. The interacting theory is formulated in space-time with a non-zero cosmological constant $\Lambda$, for instance on a $D$-dimensional anti de Sitter background. The coupling constants in a perturbative expansion of higher spin interactions are proportional to inverse powers of $\Lambda$ and thus do not admit a naive flat space-time limit. ${ }^{2}$ The theory is gauge invariant under an infinitedimensional non-Abelian higher spin gauge symmetry, which contains an $\operatorname{AdS}_{D}$ isometry group $\mathrm{SO}(D-1,2)$ as a finite dimensional subgroup. In $D=4$ and in the free field limit, the $\mathrm{SO}(3,2)$ symmetry is extended to the conformal symmetry $\mathrm{SO}(4,2)$, the latter being (spontaneously) broken by higher spin interactions. Nevertheless, it is often important to first understand the symmetries of the free theory, which are already quite nontrivial for higher spin gauge theories, and then study their implications when the interactions are switched on. The requirement that the nonlinear interaction possesses a part of, or some kind of nonlinear deformations of the original free theory, can be a good selection criterion for the allowed interaction terms. The study of one of the "hidden" symmetries of free field equations of massless higher spin fields and, in particular, the restrictions that this symmetry imposes on their correlation functions in flat and AdS spaces is the subject of this paper. In $D=4$, the hidden symmetry in question is $\operatorname{Sp}(8)$ and contains the conformal group $\mathrm{SO}(4,2)$ as a subgroup. To make the $\mathrm{Sp}(8)$ symmetry manifest, we will consider a formulation of free higher spin theory in which the conventional space-time is extended with extra coordinates in a way that is different from (or complementary to) eq. (1.1). We will call such an extension 'hyperspace' [15] to reconcile the different names given in earlier papers, such as tensorial space [16, 17] or matrix space [18].

The symmetry that we are going to explore was first observed in [19] using the following reasoning. It is well known that the group $\mathrm{SO}(3,2) \sim \mathrm{Sp}(4)$, which is the isometry group of a four-dimensional AdS space and of the conformal group in three dimensions, has a so-called singleton representation associated with a $3 d$ scalar and spinor field. According to the Flato-Fronsdal theorem [20], the $\mathrm{Sp}(4) \times \mathrm{Sp}(4)$ product of two singleton modules generates an infinite sum of massless higher spin states in $D=4$ with each spin $s$ appearing once. The integer and half-integer spin sets of these states form infinite representations of the $\mathrm{Sp}(8)$ group, which contains $\mathrm{Sp}(4) \times \mathrm{Sp}(4)$ and $\mathrm{SO}(4,2)$ as subgroups. Fronsdal observed that ten is the minimal dimension of space, which contains the four-dimensional space-time as a subspace, and in which the $\operatorname{Sp}(8)$ symmetry acts geometrically, i.e. it acts on the points of this space in a way similar to conformal transformations in flat or AdS space-time. His idea was that there should exist a theory in this $10 d$ hyperspace which, in a way alternative to that of Kaluza and Klein, would reproduce the massless higher spin field theory in the $4 d$ space-time.

\footnotetext{
${ }^{2}$ Although Vasiliev's nonlinear equations are formulated in a background independent way, their perturbative expansion is also (usually) performed around the $\mathrm{AdS}_{D}$ background which is one of the admissible vacuum solutions of these equations, see e.g. [7, 9, 12] for a review.
} 
The first explicit realization of this idea was a twistor-like superparticle model of Bandos and Lukierski [16] which, for $D=4$, possesses the generalized superconformal symmetry under $\operatorname{OSp}(1 \mid 8)$. The original motivation behind this model was not related to higher spins, but to a geometric interpretation of commuting tensorial charges of an extended supersymmetry algebra as momenta conjugate to six tensorial coordinates $y^{m n}=-y^{n m}$ $(m, n=0,1,2,3)$, which extend four space-time coordinates $x^{m}$ to the ten-dimensional hyperspace

$$
X^{\mu \nu}=X^{\nu \mu}=\frac{1}{2} x^{m} \gamma_{m}^{\mu \nu}+\frac{1}{4} y^{m n} \gamma_{m n}^{\mu \nu}, \quad \mu, \nu=1,2,3,4,
$$

where $\gamma_{m}^{\mu \nu}=\gamma_{m}^{\nu \mu}$ are four-dimensional symmetric gamma-matrices.

The higher spin content of this model was found later in [17] where the quantum states of the superparticle were shown to form an infinite tower of massless higher spin fields, and the relation of this model to the unfolded formulation was assumed. This relation was analyzed in detail in [18, 21-24].

In particular, in [21] it was demonstrated that the field equations in a super-hyperspace $\mathcal{M}_{N \mid n}$ of bosonic dimension $\frac{1}{2} n(n+1)$ and of fermionic dimension $n N$ are $\operatorname{OSp}(N \mid 2 n)$ invariant and, for $n=4$, they correspond to the unfolded higher spin field free equations in $D=4$. It has also been shown [18] that the theory possesses properties of causality and locality. A detailed analysis of free field equations in hyperspaces associated with spacetimes of dimension $D=3,4,6$ and 10 was further carried out in [24]. Two- and three-point $\operatorname{Sp}(2 n)$-invariant correlation functions of scalar and spinor fields in flat hyperspace $\mathcal{M}_{n}$ were computed in $[18,25]$. In the unfolded formalism, $\operatorname{Sp}(2 n)$-invariant multi-point functions were given in [26], that generalized four-dimensional 2- and 3-point function computations of [27]. Other aspects of the hyperspace formulation and its supersymmetrization have been considered in [15, 28-34] (see also [35]).

The results mentioned above were obtained in flat hyperspace that contains conventional Minkowski space-time as a subspace. However, also AdS (super)spaces admit the hyperspace extensions $[21,36,37]$. These are (super)group manifolds $\operatorname{OSp}(N \mid n)$. In particular, the hyperspace extension of $N=1 \mathrm{AdS}_{4}$ superspace is the supergroup $\operatorname{OSp}(1 \mid 4)$. In [38] and [23] it was shown that $\operatorname{Sp}(8)$-invariant field equations on $\operatorname{Sp}(4)$ lead to free unfolded equations for massless higher spin fields in $\mathrm{AdS}_{4}$.

In this paper, we continue the study of the dynamics of massless higher spin fields in flat and $\operatorname{Sp}(n)$ hyperspaces. In particular, exploiting the property that $\operatorname{Sp}(n)$ group manifolds are 'GL-flat' $[22,23]$, i.e. they are related to the flat hyperspace by a 'generalized conformal' (general linear) transformation, we find the explicit relation between the solutions of the $\operatorname{Sp}(2 n)$-invariant field equations in flat hyperspace and on $\operatorname{Sp}(n)$, as well as the relation between the $\operatorname{Sp}(2 n)$-invariant correlation functions of fields in these spaces. Requiring $\mathrm{Sp}(2 n)$ symmetry, we also derive the explicit form of the four-point correlation functions in these hyperspaces, which turns out to be analogous to the form of correlation functions in conformal field theories.

The paper is organized as follows. In section 2, we collect the main facts about scalar and spinor field theories on flat hyperspaces. We give an explicit form of the field equations, 
describe their $\operatorname{Sp}(2 n)$ symmetry group and review how the linearized curvatures for massless higher spin fields in the conventional flat space-time are obtained in this approach.

In section 3, we discuss the scalar and spinor field theories on $\operatorname{Sp}(n)$ group manifolds. As mentioned above, these manifolds are actually hyperspace extensions of AdS spaces, and the field equations on $\operatorname{Sp}(n)$ manifolds are deformations of the ones on flat hyperspaces, with the deformation parameter being related to the corresponding AdS radius.

In section 4, we establish a connection between the previous two sections. In particular, we show that the field equations on flat and AdS hyperspaces are related via a generalized conformal transformation of the scalar and spinor fields, similarly to the case of scalar and spinor fields on the ordinary flat and AdS spaces. The crucial tool in establishing the connection between flat and AdS hyperspaces is the $\mathrm{GL}(n)$ flatness property of $\mathrm{Sp}(n)$ group manifolds [22], which is a generalization of the conformal flatness property of conventional AdS spaces.

Section 5 is, in a certain sense, complementary to the rest of the paper. There, we show by explicit computation how the metric on a four dimensional AdS space is obtained from the $\mathrm{Sp}(4)$ hyperspace and derive the exact relation between the contraction parameter of the $\mathrm{Sp}(4)$ algebra and the radius of $\mathrm{AdS}_{4}$.

In section 6 we present computations of various correlation functions on flat and AdS hyperspaces. The two- and three- point correlation functions on flat hyperspaces were obtained previously in [25]. We follow a similar approach to derive four-point functions on flat hyperspace for bosonic and fermionic fields. Having obtained correlators on flat hyperspaces, we use the generalized conformal transformation relating the fields on flat and AdS hyperspaces in order to obtain the correlators on $\operatorname{Sp}(n)$ group manifolds.

The last section contains our conclusions and open questions for future research.

Finally, the appendix summarizes technical details that are useful for the calculations.

\section{Scalar and spinor field theory in flat hyperspace}

The points of the flat hyperspace $\mathcal{M}_{n}$ are parametrized by symmetric matrix coordinates $X^{\mu \nu}=X^{\nu \mu}(\mu, \nu=1, \ldots, n)$. The linear symmetries of $\mathcal{M}_{n}$ are rigid translations and $\mathrm{GL}(n)$ rotations generated, respectively, by

$$
P_{\mu \nu}=-i \frac{\partial}{\partial X^{\mu \nu}} \equiv-i \partial_{\mu \nu}, \quad\left[P_{\mu \nu}, P_{\rho \lambda}\right]=0
$$

and

$$
L_{\nu}{ }^{\mu}=-2 i X^{\mu \rho} \partial_{\rho \nu}, \quad\left[L_{\nu}{ }^{\mu}, L_{\lambda}{ }^{\rho}\right]=i\left(\delta_{\lambda}^{\mu} L^{\rho}{ }_{\nu}-\delta_{\nu}^{\rho} L_{\lambda}^{\mu}\right),
$$

where, by definition,

$$
\frac{\partial X^{\mu \nu}}{\partial X^{\rho \lambda}}=\frac{1}{2}\left(\delta_{\rho}^{\mu} \delta_{\lambda}^{\nu}+\delta_{\rho}^{\nu} \delta_{\lambda}^{\mu}\right)
$$

Under (2.1) and (2.2) the hyperspace coordinates are transformed as follows

$$
\delta X^{\mu \nu}=i\left(a^{\rho \lambda} P_{\rho \lambda}+g_{\rho}{ }^{\lambda} L_{\lambda}{ }^{\rho}\right) X^{\mu \nu}=a^{\mu \nu}+\left(X^{\mu \rho} g_{\rho}{ }^{\nu}+X^{\nu \rho} g_{\rho}{ }^{\mu}\right),
$$

where $a^{\mu \nu}=a^{\nu \mu}$ and $g_{\mu}{ }^{\nu}$ are arbitrary constant parameters. 
These symmetries are the hyperspace counterparts of the conventional Poincaré translations, Lorentz rotations and dilatations of Minkowski space-time. Generalized Lorentz rotations are generated by traceless operators $L_{\mu}{ }^{\nu}-\frac{1}{n} \delta_{\mu}^{\nu} L_{\lambda}{ }^{\lambda}$, forming the $\mathrm{SL}(n)$-algebra, whereas dilatations are generated by the trace of $L_{\mu}{ }^{\nu}$.

One may enlarge these transformations by considering generalized conformal boosts

$$
K^{\mu \nu}=i X^{\mu \rho} X^{\nu \lambda} \partial_{\rho \lambda}, \quad\left[K^{\mu \nu}, K^{\rho \lambda}\right]=0
$$

so that the total transformation of $X^{\mu \nu}$ becomes

$$
\begin{aligned}
\delta X^{\mu \nu} & =i\left(a^{\rho \lambda} P_{\rho \lambda}+g_{\rho}{ }^{\lambda} L_{\lambda}{ }^{\rho}+k_{\rho \lambda} K^{\rho \lambda}\right) X^{\mu \nu} \\
& =a^{\mu \nu}+\left(X^{\mu \rho} g_{\rho}{ }^{\nu}+X^{\nu \rho} g_{\rho}{ }^{\mu}\right)-X^{\mu \rho} k_{\rho \lambda} X^{\lambda \nu}
\end{aligned}
$$

where $k_{\mu \nu}=k_{\nu \mu}$ are constant parameters of the boosts.

The generators (2.1), (2.2) and (2.5) form the $\operatorname{Sp}(2 n)$ algebra which plays the role of a generalized conformal symmetry in the hyperspace

$$
\begin{aligned}
& {\left[P_{\mu \nu}, P_{\rho \lambda}\right]=0, \quad\left[K^{\mu \nu}, K^{\rho \lambda}\right]=0, \quad\left[{L_{\nu}}^{\mu},{L_{\lambda}}^{\rho}\right]=i\left(\delta_{\lambda}^{\mu} L_{\nu}{ }^{\rho}-\delta_{\nu}^{\rho} L_{\lambda}{ }^{\mu}\right),} \\
& {\left[P_{\mu \nu}, L_{\lambda}^{\rho}\right]=-i\left(\delta_{\mu}^{\rho} P_{\nu \lambda}+\delta_{\nu}^{\rho} P_{\mu \lambda}\right), \quad\left[K^{\mu \nu}, L_{\lambda}{ }^{\rho}\right]=i\left(\delta_{\lambda}^{\mu} K^{\nu \rho}+\delta_{\lambda}^{\nu} K^{\mu \rho}\right),} \\
& {\left[P_{\mu \nu}, K^{\lambda \rho}\right]=\frac{i}{4}\left(\delta_{\mu}^{\rho} L_{\nu}{ }^{\lambda}+\delta_{\nu}^{\rho} L_{\mu}{ }^{\lambda}+\delta_{\mu}^{\lambda} L_{\nu}{ }^{\rho}+\delta_{\nu}^{\lambda} L_{\mu}{ }^{\rho}\right) .}
\end{aligned}
$$

From the structure of this algebra, one can see that the flat hyperspace $\mathcal{M}_{n}$ can be realized as a coset manifold associated with the translations $P=\frac{\mathrm{Sp}(2 n)}{K \otimes \operatorname{SL}(n)}$ where $K \otimes \mathrm{SL}(n)$ is the semi-direct product of the general linear group and the boosts $K_{\mu \nu}$.

In the case $n=4$, which is related to the higher spin theory in $D=4$ (see eq. (1.2)), the generalized conformal symmetry of $\mathcal{M}_{4}$ is $\mathrm{Sp}(8)$. As was previously shown in [21], the dynamics of the free higher spin fields in flat $D=4$ space-time is encoded into two hyperfields. A scalar field $b(X)$ incorporates the field strengths of the $4 d$ fields of integer spins and a spinor field $f_{\mu}(X)$ incorporates the half-integer spin field strengths. ${ }^{3}$ They satisfy the following field equations [21]

$$
\begin{gathered}
\left(\partial_{\mu \nu} \partial_{\rho \lambda}-\partial_{\mu \rho} \partial_{\nu \lambda}\right) b(X)=0 \\
\partial_{\mu \nu} f_{\rho}(X)-\partial_{\mu \rho} f_{\nu}(X)=0
\end{gathered}
$$

Note that, in the above equations, there is no contraction of indices, implying that $a$ priori we do not endow the hyperspace with a metric structure. As we will see below, the metric structure will appear upon reduction of these equations to the physical space-time by expanding the tensorial coordinates in the basis of the gamma-matrices as in eq. (1.2). The Minkowski metric then appears as a consequence of the use of the Clifford algebra $\left\{\gamma^{m}, \gamma^{n}\right\}=2 \eta^{m n}$.

\footnotetext{
${ }^{3}$ We call the field $f_{\mu}(X)$ spinor since in the physically interesting cases the index $\mu$ is associated with a spinor representation of the Lorenz group in the $D$-dimensional subspace-time of the hyperspace.
} 
In any $\mathcal{M}_{n}$, the equations (2.8) and (2.9) are invariant under the $\operatorname{Sp}(2 n)$ transformations (2.6), provided that the fields transform as follows

$$
\begin{aligned}
\delta b(X)= & -\left(a^{\mu \nu} \partial_{\mu \nu}+\frac{1}{2} g_{\mu}{ }^{\mu}+2 g_{\nu}{ }^{\mu} X^{\nu \rho} \partial_{\mu \rho}-k_{\mu \nu}\left(\frac{1}{2} X^{\mu \nu}+X^{\mu \rho} X^{\nu \lambda} \partial_{\rho \lambda}\right)\right) b(X), \\
\delta f_{\rho}(X)= & -\left(a^{\mu \nu} \partial_{\mu \nu}+\frac{1}{2} g_{\mu}{ }^{\mu}+2 g_{\nu}{ }^{\mu} X^{\nu \lambda} \partial_{\mu \lambda}-k_{\mu \nu}\left(\frac{1}{2} X^{\mu \nu}+X^{\mu \tau} X^{\nu \lambda} \partial_{\tau \lambda}\right)\right) f_{\rho}(X) \\
& -\left(g_{\rho}{ }^{\nu}-k_{\lambda \rho} X^{\lambda \nu}\right) f_{\nu}(X) .
\end{aligned}
$$

Note that these variations contain the term $\frac{1}{2}\left(g_{\mu}{ }^{\mu}-k_{\mu \nu} X^{\mu \nu}\right)$, implying that the fields have the canonical conformal weight $1 / 2$. A natural generalization of these transformations for fields of a generic conformal weight $\Delta$ is

$$
\begin{aligned}
\delta b(X)= & -\left(a^{\mu \nu} \partial_{\mu \nu}+\Delta\left(g_{\mu}{ }^{\mu}-k_{\mu \nu} X^{\mu \nu}\right)+2 g_{\nu}{ }^{\mu} X^{\nu \rho} \partial_{\mu \rho}-k_{\mu \nu} X^{\mu \rho} X^{\nu \lambda} \partial_{\rho \lambda}\right) b(X), \\
\delta f_{\rho}(X)= & -\left(a^{\mu \nu} \partial_{\mu \nu}+\Delta\left(g_{\mu}{ }^{\mu}-k_{\mu \nu} X^{\mu \nu}\right)+2 g_{\nu}{ }^{\mu} X^{\nu \lambda} \partial_{\mu \lambda}-k_{\mu \nu} X^{\mu \tau} X^{\nu \lambda} \partial_{\tau \lambda}\right) f_{\rho}(X) \\
& -\left(g_{\rho}{ }^{\nu}-k_{\lambda \rho} X^{\lambda \nu}\right) f_{\nu}(X) .
\end{aligned}
$$

In the case of $n=2$, the hyperspace $\mathcal{M}_{2}$ is just the ordinary $D=3$ Minkowski space parametrized by $X^{\mu \nu}=x^{m} \gamma_{m}^{\mu \nu}(m=0,1,2)$ and, as one may easily check, eqs. (2.8) and (2.9) reduce, respectively, to the Klein-Gordon equation for the massless scalar $b(x)$ and the massless Dirac equation for the Majorana spinor $f_{\mu}(x)$, which are conformally invariant.

In the case of $\mathcal{M}_{4}$, eqs. (2.8) and (2.9) produce in $D=4$ the conformally invariant set of Bianchi identities and equations of motion for linearized field strengths of the massless fields of all spins $s=0, \frac{1}{2}, 1,2, \ldots, \infty$, while the cases $n=8$ and $n=16$ describe conformally invariant higher spin fields whose field strengths are self-dual, respectively, in $D=6$ and $D=10$, as was shown in detail in [24].

For instance, to obtain the higher spin field equations from (2.8) and (2.9) in the four-dimensional case, one expands $b(X)$ and $f_{\mu}(X)$ in powers of the extra coordinates $y^{m n}=-y^{n m}$, eq. (1.2), as follows

$$
\begin{aligned}
b\left(x^{l}, y^{m n}\right)= & \phi(x)+y^{m_{1} n_{1}} F_{m_{1} n_{1}}(x)+y^{m_{1} n_{1}} y^{m_{2} n_{2}}\left[R_{m_{1} n_{1}, m_{2} n_{2}}(x)-\frac{1}{2} \eta_{m_{1} m_{2}} \partial_{n_{1}} \partial_{n_{2}} \phi(x)\right] \\
& +\sum_{s=3}^{\infty} y^{m_{1} n_{1}} \cdots y^{m_{s} n_{s}}\left[R_{m_{1} n_{1}, \cdots, m_{s} n_{s}}(x)+\cdots\right]
\end{aligned}
$$$$
f^{\rho}\left(x^{l}, y^{m n}\right)=\psi^{\rho}(x)+y^{m_{1} n_{1}}\left[R_{m_{1} n_{1}}^{\rho}(x)-\frac{1}{2} \partial_{m_{1}}\left(\gamma_{n_{1}} \psi\right)^{\rho}\right]
$$$$
+\sum_{s=\frac{5}{2}}^{\infty} y^{m_{1} n_{1}} \cdots y^{m_{s-\frac{1}{2}} n_{s-\frac{1}{2}}}\left[R_{m_{1} n_{1}, \cdots, m_{s-\frac{1}{2}} n_{s-\frac{1}{2}}}^{\rho}(x)+\cdots\right] .
$$

In (2.14), $\phi(x)$ and $\psi^{\rho}(x)$ are a scalar and a spinor field, respectively, $F_{m_{1} n_{1}}(x)$ is the Maxwell field strength, $R_{m_{1} n_{1}, m_{2} n_{2}}(x)$ is the curvature tensor of linearized gravity, $R_{m_{1} n_{1}}^{\rho}(x)$ 
is the Rarita-Schwinger field strength and other terms in the series stand for generalized Riemann curvatures of spin- $s$ fields ${ }^{4}$ (that also contain contributions of derivatives of the fields of lower spin denoted by dots, as in the case of the Rarita-Schwinger and gravity fields).

Substituting the expressions (2.14) into eqs. (2.8) and (2.9), and rewriting the derivatives explicitly as

$$
\partial_{\mu \nu}=\frac{1}{2}\left(\gamma_{\mu \nu}^{m} \frac{\partial}{\partial x^{m}}+\gamma_{\mu \nu}^{m n} \frac{\partial}{\partial y^{m n}}\right)
$$

one finds that the scalar and the spinor field satisfy, respectively, the Klein-Gordon and the Dirac equations, while the higher spin field curvatures satisfy the Bianchi identities

$$
R_{\left[m_{1} n_{1}, m_{2}\right] n_{2}, \cdots, m_{s} n_{s}}=0, \quad \partial_{\left[l_{1}\right.} R_{\left.m_{1} n_{1}\right], m_{2} n_{2}, \cdots, m_{s} n_{s}}=0,
$$

and the linearized higher spin field equations

$$
R_{n_{1}, m n_{2}, m_{3} n_{3}, \cdots, m_{s} n_{s}}^{m}=0, \quad\left(\gamma^{m_{1}} R\right)_{m_{1} n_{1}, m_{2} n_{2}, \cdots, m_{s-\frac{1}{2}} n_{s-\frac{1}{2}}}^{\mu}=0 .
$$

To the best of our knowledge, equations similar to (2.16) and (2.17) first appeared in the Weinberg paper [39]. In [40], in a 'symmetric' Young-tableaux convention, higher spin curvatures (and generalized Christoffel symbols) were constructed as $s$-derivatives of Fronsdal [1] potentials of spin-s. In the same form as (2.16) and (2.17) the curvature equations were given e.g. in [41], and in [42, 43] it was shown that these equations for integer-spin curvatures are equivalent to s-derivative equations on unconstrained spin-s potentials and are invariant under unconstrained local higher-spin symmetries. These equations, in turn, are reduced (upon a partial gauge fixing) to the second-order Fronsdal equations [43]. For half-integer higher-spin fields these results were generalized in [24] to which we refer the reader for further details on field theories in flat hyperspaces and proceed to discuss hyperspace field theories related to higher spin fields in AdS.

\section{Scalar and spinor field theory on the group manifold $\operatorname{Sp}(n)$}

As was noticed in $[36,37]$ and [21], the hyperspace extension of the $\mathrm{AdS}_{4}$ space is the group manifold $\mathrm{SO}(3,2) \sim \mathrm{Sp}(4)$ which contains the $\mathrm{AdS}_{4}=\frac{\mathrm{SO}(3,2)}{\mathrm{SO}(3,1)}$ symmetric space as a coset subspace of maximal dimension. For $n>4$, an $\operatorname{AdS}_{d}$ space is also a subspace of $\operatorname{Sp}(n)$ but is no longer the maximal coset of this group.

Before generalizing the field equations (2.8) and (2.9) to the $\operatorname{Sp}(n)$ case, let us recall the basic group-theoretical and geometric properties of the $\operatorname{Sp}(n)$ group manifold.

The group $\operatorname{Sp}(n)$ is generated by $n \times n$ symmetric matrices $M_{\alpha \beta}$ forming the algebra

$$
\left[M_{\alpha \beta}, M_{\gamma \delta}\right]=-\frac{i \xi}{2}\left[C_{\gamma(\alpha} M_{\beta) \delta}+C_{\delta(\alpha} M_{\beta) \gamma}\right], \quad \alpha, \beta=1, \ldots, n,
$$

where $C_{\alpha \beta}=-C_{\beta \alpha}$ is an $\operatorname{Sp}(n)$-invariant symplectic metric and the parameter $\xi$ has the inverse dimension of length. As will be shown explicitly in section 5 , the parameter $\xi$ is

\footnotetext{
${ }^{4}$ The pairs of the indices separated by the commas are antisymmetrized.
} 
related to the radius of the AdS space. Its presence in the $\operatorname{Sp}(n)$ algebra allows one to perform (at $\xi \rightarrow 0$ ) its contraction to the algebra of translations $M_{\alpha \beta} \rightarrow P_{\alpha \beta}$ (2.1) of the flat $\mathcal{M}_{n}$ hyperspace.

As a group manifold, $\operatorname{Sp}(n)$ is the coset $\operatorname{Sp}(n)_{L} \times \operatorname{Sp}(n)_{R} / \operatorname{Sp}(n)$ which has the isometry group $\operatorname{Sp}(n)_{L} \times \operatorname{Sp}(n)_{R}$, the latter being the subgroup of $\operatorname{Sp}(2 n)$ generated by

$$
M_{\alpha \beta}^{L}=P_{\alpha \beta}-\frac{\xi^{2}}{16} K_{\alpha \beta}-\frac{\xi}{4} L_{(\alpha \beta)} \quad M_{\alpha \beta}^{R}=P_{\alpha \beta}-\frac{\xi^{2}}{16} K_{\alpha \beta}+\frac{\xi}{4} L_{(\alpha \beta)},
$$

as one may see from the structure of the $\operatorname{Sp}(2 n)$ algebra (2.7). In (3.2), $K_{\alpha \beta}=C_{\alpha \gamma} C_{\beta \delta} K^{\gamma \delta}$ and $L_{(\alpha \beta)}=\frac{1}{2}\left(L_{\alpha}^{\gamma} C_{\gamma \beta}+L_{\beta}^{\gamma} C_{\gamma \alpha}\right)$. The latter generate the diagonal $\operatorname{Sp}(n)$ subalgebra of $\operatorname{Sp}(n)_{L} \times \operatorname{Sp}(n)_{R}$. This algebraic structure implies that $\operatorname{Sp}(n)$ can also be realized as a coset manifold of $\mathrm{Sp}(2 n)$ associated with the generators $P-\xi^{2} K=\frac{\mathrm{Sp}(2 n)}{\mathrm{SL}(n) \otimes K}$. This coset is apparently different from the $\operatorname{Sp}(2 n)$ coset realization of the flat hyperspace $\mathcal{M}_{n}$ discussed in the previous section, but it implies that the two manifolds can actually be related to each other by an $\operatorname{Sp}(2 n)$ transformation in a way similar to the conformal flatness of the conventional Minkowski and AdS space. This property will be discussed in detail in the next section.

The $\operatorname{Sp}(n)$ group element $\mathcal{O}(X)$, parametrized by the coordinates $X^{\mu \nu}$, defines Cartan forms $\Omega^{\alpha \beta}(X)$

$$
\mathcal{O}^{-1} d \mathcal{O}=\Omega^{\alpha \beta} M_{\alpha \beta}=d X^{\mu \nu} E_{\mu \nu}^{\alpha \beta}(X) M_{\alpha \beta} .
$$

The Cartan forms encode the vielbeine and the spin connections which characterize a geometry of $\operatorname{Sp}(n)$. In eq. (3.3) we distinguish the flat tangent-space basis on $\operatorname{Sp}(n)$, labeled by the letters $\alpha, \beta, \ldots$ (from the beginning of the Greek alphabet), from the curved world basis associated with $X^{\mu \nu}$, labeled by the letters $\mu, \nu, \ldots$ (from the middle of the Greek alphabet).

By construction, the Cartan forms (3.3) obey the Maurer-Cartan equations, which according to the algebra (3.1) have the form

$$
d \Omega^{\alpha \beta}+\frac{\xi}{2} \Omega^{\alpha \gamma} \wedge \Omega_{\gamma}{ }^{\beta}=0
$$

where the indices are lowered and raised by $C_{\alpha \beta}$ and $C^{\alpha \beta}$ as in eq. (A.2).

As in the general case of the group manifolds, one can define a geometry of $\operatorname{Sp}(n)$ to be flat with non-trivial torsion, or to have zero torsion and constant curvature.

In the zero-curvature geometry one chooses the spin connection to be zero and a local tangent-space basis to be formed by the vielbeine $E^{\alpha \beta} \equiv \Omega^{\alpha \beta}=d X^{\mu \nu} E_{\mu \nu}^{\alpha \beta}(X)$. From the Maurer-Cartan equations it then follows that the $\operatorname{Sp}(n)$ torsion is

$$
T^{\alpha \beta}=d E^{\alpha \beta}=-\frac{\xi}{2} E^{\alpha \gamma} \wedge E_{\gamma}^{\beta} .
$$

The covariant derivatives associated with this geometry are constructed with the use of the inverse vielbeine

$$
\nabla_{\alpha \beta}=E_{\alpha \beta}^{\mu \nu}(X) \partial_{\mu \nu}, \quad E_{\alpha \beta}^{\mu \nu} E_{\mu \nu}^{\gamma \delta}=\frac{1}{2}\left(\delta_{\alpha}^{\gamma} \delta_{\beta}^{\delta}+\delta_{\alpha}^{\delta} \delta_{\beta}^{\gamma}\right),
$$


and form the $\operatorname{Sp}(n)$-algebra

$$
\left[\nabla_{\alpha \beta}, \nabla_{\gamma \delta}\right]=\frac{\xi}{4}\left(C_{\alpha \gamma} \nabla_{\beta \delta}+C_{\alpha \delta} \nabla_{\beta \gamma}+C_{\beta \gamma} \nabla_{\alpha \delta}+C_{\beta \delta} \nabla_{\alpha \gamma}\right)
$$

On the other hand, one can interpret (3.4) as the torsion-free condition for the $\operatorname{Sp}(n)$ geometry with curvature, described by the vielbein $E^{\alpha \beta}$ and the connection $\omega_{\alpha}^{\beta}$ defined as follows

$$
E^{\alpha \beta}=\Omega^{\alpha \beta}, \quad \omega_{\alpha}^{\beta}=\frac{\xi}{4} \Omega_{\alpha}{ }^{\beta} .
$$

The zero-torsion condition takes the form

$$
T^{\alpha \beta}=D E^{\alpha \beta}=d E^{\alpha \beta}+E^{\alpha \gamma} \wedge \omega_{\gamma}^{\beta}+E^{\beta \gamma} \wedge \omega_{\gamma}^{\alpha}=0
$$

and the $\operatorname{Sp}(n)$ curvature is

$$
R_{\alpha}{ }^{\beta}=d \omega_{\alpha}{ }^{\beta}+\omega_{\alpha}^{\gamma} \wedge \omega_{\gamma}{ }^{\beta}=-\frac{\xi^{2}}{16} E_{\alpha}^{\gamma} \wedge E_{\gamma}{ }^{\beta}
$$

The covariant differential

$$
D=E^{\alpha \beta} D_{\alpha \beta}=E^{\alpha \beta} \nabla_{\alpha \beta}+\omega,
$$

acts on the contravariant and covariant spinors $F^{\alpha}$ and $F_{\alpha}$ as follows

$$
\begin{aligned}
& D F^{\gamma}=d F^{\gamma}+F^{\gamma} \omega_{\gamma}^{\alpha}=E^{\alpha \beta}\left(\nabla_{\alpha \beta} \delta_{\delta}^{\gamma}+\frac{\xi}{8} C_{\alpha \delta} \delta_{\beta}^{\gamma}+\frac{\xi}{8} C_{\beta \delta} \delta_{\alpha}^{\gamma}\right) F^{\delta}, \\
& D F_{\gamma}=d F_{\gamma}-\omega_{\alpha}^{\gamma} F_{\gamma}=E^{\alpha \beta}\left(\nabla_{\alpha \beta} \delta_{\gamma}^{\delta}-\frac{\xi}{8} C_{\alpha \gamma} \delta_{\beta}^{\delta}-\frac{\xi}{8} C_{\beta \gamma} \delta_{\alpha}{ }^{\delta}\right) F_{\delta} .
\end{aligned}
$$

With the use of the zero-curvature covariant derivatives (3.6), the equations of motion of a bosonic field $B(x)$ and a fermionic field $F_{\alpha}(X)$, generalizing the flat hyperspace field equations (2.8) and (2.9) to the $\operatorname{Sp}(n)$ group manifold, have the following form [23]

$$
\begin{array}{r}
\left(\nabla_{\alpha \beta} \nabla_{\gamma \delta}-\nabla_{\alpha \gamma} \nabla_{\beta \delta}\right) B \\
-\frac{\xi}{8}\left(C_{\alpha \gamma} \nabla_{\beta \delta}-C_{\alpha \beta} \nabla_{\gamma \delta}+C_{\beta \delta} \nabla_{\alpha \gamma}-C_{\gamma \delta} \nabla_{\alpha \beta}+2 C_{\beta \gamma} \nabla_{\alpha \delta}\right) B \\
-\left(\frac{\xi}{8}\right)^{2}\left(C_{\alpha \gamma} C_{\beta \delta}-C_{\alpha \beta} C_{\gamma \delta}+2 C_{\beta \gamma} C_{\alpha \delta}\right) B=0 \\
\nabla_{\alpha \beta} F_{\gamma}-\nabla_{\alpha \gamma} F_{\beta}+\frac{\xi}{8}\left(C_{\gamma \alpha} F_{\beta}-C_{\beta \alpha} F_{\gamma}+2 C_{\gamma \beta} F_{\alpha}\right)=0 .
\end{array}
$$

In the basis of the zero-torsion covariant derivatives (3.13), the fermionic equation simplifies to

$$
D_{\alpha \beta} F_{\gamma}-D_{\alpha \gamma} F_{\beta}=0,
$$

while the bosonic equation takes the form

$$
\left(D_{\alpha \beta} D_{\gamma \delta}-D_{\alpha \gamma} D_{\beta \delta}\right) B-\left(\frac{\xi}{8}\right)^{2}\left(C_{\alpha \gamma} C_{\beta \delta}-C_{\alpha \beta} C_{\gamma \delta}+2 C_{\beta \gamma} C_{\alpha \delta}\right) B=0 .
$$


Equations (3.14)-(3.17) are $\operatorname{Sp}(2 n)$-invariant. This fact stems from the origin of these equations from the quantization of a corresponding $\operatorname{Sp}(2 n)$-invariant particle model $[22,23]$. We will explicitly show this below using the generalized conformal flatness of the $\operatorname{Sp}(n)$ manifold.

As we have mentioned, the flat hyperspace and the $\operatorname{Sp}(n)$ group manifold can be realized as different cosets of their generalized conformal group $\operatorname{Sp}(2 n)$. This prompts one to ask whether their geometries, as well as the solutions of the scalar and spinor field equations in flat and $\operatorname{Sp}(n)$ hyperspace, can locally be related by a generalized conformal transformation in a way similar to the conformally flat cases of conventional Minkowski and AdS spaces.

The answer to this question turns out to be positive. In order to explicitly demonstrate the connection between the two systems we will explore a special property of the $\operatorname{Sp}(n)$ group manifolds found in [22] and called 'GL-flatness', where 'GL' stands for 'general linear' or 'generalized conformal' flatness.

\section{GL-flatness of $\operatorname{Sp}(n)$ group manifolds and the relation between the field equations in flat and $\operatorname{Sp}(n)$ hyperspaces}

By GL-flatness of the $\operatorname{Sp}(n)$ manifold we mean that, in a local coordinate basis associated with $X^{\alpha \beta}$, the covariant derivatives $\nabla_{\alpha \beta}(3.6)$ satisfying the $\operatorname{Sp}(n)$ algebra (3.7) take a very simple form

$$
\nabla_{\alpha \beta}=G_{\alpha}^{-1 \mu}(X) G_{\beta}^{-1 \nu}(X) \partial_{\mu \nu},
$$

where $G_{\alpha}^{-1 \mu}(X)$ is a matrix which depends linearly on $X_{\alpha}{ }^{\mu}$

$$
G_{\alpha}^{-1 \mu}(X)=\delta_{\alpha}^{\mu}+\frac{\xi}{4} X_{\alpha}{ }^{\mu}
$$

The corresponding $\operatorname{Sp}(n)$ Cartan forms $\Omega^{\alpha \beta}$ are

$$
\Omega^{\alpha \beta}=d X^{\mu \nu} G_{\mu}^{\alpha}(X) G_{\nu}^{\beta}(X),
$$

where the matrix $G_{\mu}{ }^{\alpha}(X)$ is inverse of $G_{\alpha}^{-1 \mu}(X)^{5}$ and has the following form

$$
G_{\mu}^{\alpha}(X)=\delta_{\mu}^{\alpha}+\sum_{k=1}^{\infty}\left(-\frac{\xi}{4}\right)^{k}\left(X^{k}\right)_{\mu}^{\alpha},
$$

where $\left(X^{k}\right)_{\mu}{ }^{\alpha}$ stands for the product of the $k$ matrices $X_{\mu}{ }^{\alpha}$. Note that the possibility of representing the Cartan forms in the form (4.3) is a particular feature of the $\operatorname{Sp}(n)$ group manifold since, in general, it is not possible to decompose the components of the Cartan form into a "direct product" of components of some matrix $G_{\mu}{ }^{\alpha}$.

GL-flatness implies that the $\operatorname{Sp}(n)$ Cartan forms and the covariant derivatives can be obtained from the flat hyperspace ones by a transformation in the group $\operatorname{GL}(n) \subset \operatorname{Sp}(2 n)$ involving the matrix $G_{\mu}{ }^{\alpha}$ and its inverse.

\footnotetext{
${ }^{5}$ Here we follow the notation of [22] in which the matrix $G_{\beta}{ }^{\alpha}(X)$ was introduced first and then $G_{\alpha}^{-1 \mu}(X)$ was derived as its inverse.
} 
The matrices $G_{\alpha}^{-1 \mu}(X)$ and $G_{\mu}{ }^{\alpha}(X)$ satisfy the following identities

$$
\begin{aligned}
\partial_{\mu \nu} G^{-1 \alpha \beta} & =\frac{\xi}{8}\left(\delta_{\mu}^{\alpha} \delta_{\nu}^{\beta}+\delta_{\mu}^{\beta} \delta_{\nu}^{\alpha}\right), \\
\partial_{\mu \nu} G_{\rho}{ }^{\sigma} & =\frac{\xi}{8}\left(G_{\rho \mu} G_{\nu}{ }^{\sigma}+G_{\rho \nu} G_{\mu}{ }^{\sigma}\right), \\
d G_{\rho}{ }^{\sigma} & =\frac{\xi}{4}\left(\Omega_{\rho}{ }^{\sigma}+2 G_{\rho}{ }^{\mu} \Omega_{\mu}{ }^{\sigma}\right), \\
\partial_{\mu \nu} \sqrt{\operatorname{det} G} & =\frac{\xi}{16} \sqrt{\operatorname{det} G}\left(G_{\mu \nu}+G_{\nu \mu}\right), \\
\partial_{\mu \nu}(\operatorname{det} G)^{-\frac{1}{2}} & =-\frac{\xi}{16}(\operatorname{det} G)^{-\frac{1}{2}}\left(G_{\mu \nu}+G_{\nu \mu}\right), \\
\partial_{\mu \nu}(\operatorname{det} G)^{-\Delta} & =-\frac{\xi \Delta}{8}(\operatorname{det} G)^{-\Delta}\left(G_{\mu \nu}+G_{\nu \mu}\right), \\
G_{\alpha \lambda}(X) G_{\beta}{ }^{\lambda}(X) & =G_{[\alpha \beta]}(X) \equiv \frac{1}{2}\left(G_{\alpha \beta}(X)-G_{\beta \alpha}(X)\right) .
\end{aligned}
$$

These identities can be used to check that equation (3.4) is indeed solved by (4.3)-(4.4) and that the fields $B(X)$ and $F_{\alpha}(X)$ satisfying equations (3.14)-(3.17) are related to the fields $b(X)$ and $f_{\mu}(X)$ satisfying the flat hyperspace equations (2.8)-(2.9) as follows

$$
\begin{aligned}
B(X) & =(\operatorname{det} G)^{-\frac{1}{2}} b(X), \\
F_{\alpha}(X) & =(\operatorname{det} G)^{-\frac{1}{2}} G_{\alpha}^{-1 \mu} f_{\mu}(X) .
\end{aligned}
$$

These relations are similar to the relations between the conformally invariant scalar and spinor equations in the conventional flat and AdS spaces and reduce to them in the case of $n=2, D=3$.

\section{1 $\operatorname{Sp}(2 n)$ transformations of the fields on $\operatorname{Sp}(n)$}

Using relations (4.12), (4.13), and the $\mathrm{Sp}(2 n)$ transformations (2.10)-(2.13) of the bosonic and fermionic fields in flat hyperspace, it is straightforward to derive the $\operatorname{Sp}(2 n)$ transformations of the fields on $\operatorname{Sp}(2 n)$.

Using the relation between the fields of weight $\Delta=\frac{1}{2}$ on flat hyperspace and on $\operatorname{Sp}(n)$ group manifold (4.12) we have the following relation between the $\operatorname{Sp}(2 n)$ transformations of the wight- $\frac{1}{2}$ fields on $\operatorname{Sp}(n)$ and in flat hyperspace

$$
\begin{aligned}
\delta B(X) & =(\operatorname{det} G)^{-\frac{1}{2}} \delta b, \\
\delta F_{\alpha} & =(\operatorname{det} G)^{-\frac{1}{2}} G_{\alpha}^{-1 \mu} \delta f_{\mu} .
\end{aligned}
$$

Note that in the above expressions the matrix $G_{\alpha}{ }^{\mu}$ is not varied since it is form-invariant, i.e. $G\left(X^{\prime}\right)$ has the same form as $G(X)$. 
Then, in view of eq. (4.9) the $\operatorname{Sp}(n)$-variations of $B(X)$ and $F_{\alpha}(X)$ have the following form

$$
\begin{aligned}
\delta B(X)= & -\left(a^{\alpha \beta} \mathcal{D}_{\alpha \beta}+\frac{1}{2}\left(g_{\alpha}{ }^{\alpha}-k_{\alpha \beta} X^{\alpha \beta}\right)+2 g_{\beta}{ }^{\alpha} X^{\beta \gamma} \mathcal{D}_{\alpha \gamma}-k_{\alpha \beta} X^{\alpha \gamma} X^{\beta \delta} \mathcal{D}_{\gamma \delta}\right) B(X), \\
\delta F_{\sigma}(X)= & -\left(a^{\alpha \beta} \mathcal{D}_{\alpha \beta}+\frac{1}{2}\left(g_{\alpha}{ }^{\alpha}-k_{\alpha \beta} X^{\alpha \beta}\right)+2 g_{\beta}{ }^{\alpha} X^{\beta \gamma} \mathcal{D}_{\alpha \gamma}-k_{\alpha \beta} X^{\alpha \gamma} X^{\beta \delta} \mathcal{D}_{\gamma \delta}\right) F_{\sigma}(X), \\
& -\left(g_{\sigma}{ }^{\beta}-k_{\sigma \alpha} X^{\alpha \beta}\right) F_{\beta}(X),
\end{aligned}
$$

where the derivative $\mathcal{D}_{\alpha \beta}$ is defined as

$$
\mathcal{D}_{\alpha \beta}=\partial_{\alpha \beta}+\frac{\xi}{16}\left(G_{\alpha \beta}+G_{\beta \alpha}\right) .
$$

Using (4.6) one can check that these derivatives commute with each other $\left[\mathcal{D}_{\alpha \beta}, \mathcal{D}_{\gamma \delta}\right]=0$ just as in the flat case.

Let us note that the relation between the flat and $\operatorname{Sp}(n)$ hyperfields of an arbitrary weight $\Delta$ and the form of the corresponding $\operatorname{Sp}(2 n)$ transformations require additional study since to this end one should know the form of $\mathrm{Sp}(2 n)$-invariant equations satisfied by these fields. In this respect, the results of $[28,44]$ on higher-rank hyperfields and currents can be useful. This issue will be addressed elsewhere.

\section{$5 \quad \mathrm{AdS}_{4}$ metric}

Before considering correlation functions, let us first demonstrate the connection between the $\mathrm{Sp}(4)$ group manifold and $\mathrm{AdS}_{4}$ space explicitly in the GL-flat basis (4.3), (4.4). In order to do so, we shall compute an explicit form of the $x^{m}$-dependent part of the metric on the $\mathrm{Sp}(4)$ group manifold in the $\mathrm{GL}(4)$ flat parametrization and prove that it corresponds to a specific parametrization of the $\mathrm{AdS}_{4}$ metric. In other words, we have to evaluate the expression

$$
\Omega^{\alpha \beta}\left(x^{m}\right)=\frac{1}{2} d x^{m}\left(\gamma_{m}\right)^{\delta \sigma} G_{\delta}{ }^{\alpha} G_{\sigma}{ }^{\beta}=\frac{1}{2} d x^{m} e_{m}^{a}\left(\gamma_{a}\right)^{\alpha \beta}+\frac{1}{4} d x^{m} \omega_{m}^{a b}\left(\gamma_{a b}\right)^{\alpha \beta},
$$

where the dependence of the matrices $X^{\alpha \beta}$ on the coordinates $y^{m n}$ (see eq. (1.2)) is discarded, i.e. $X_{\alpha}{ }^{\beta}=\frac{1}{2} x^{n}\left(\gamma_{n}\right)_{\alpha}{ }^{\beta}$. Denoting $x^{2}=x^{m} x^{n} \eta_{m n}$ and $x_{m}=\eta_{m n} x^{n}$ and, using the explicit form (4.4) of $G_{\mu}{ }^{\alpha}(X)$, one obtains

$$
\Omega^{\alpha \beta}(x)=\frac{1}{2} \frac{d x^{m}}{\left[1-\left(\frac{\xi}{8}\right)^{2} x^{2}\right]^{2}}\left[\left(\gamma_{\ell}\right)^{\alpha \beta}\left(\left[1+\left(\frac{\xi}{8}\right)^{2} x^{2}\right] \delta_{m}^{\ell}-2\left(\frac{\xi}{8}\right)^{2} \eta_{m n} x^{n} x^{\ell}\right)-\frac{\xi}{4} x^{n}\left(\gamma_{m n}\right)^{\alpha \beta}\right] .
$$

Hence, the vierbein and spin-connection take the form:

$$
\begin{aligned}
e_{m}^{a} & =\frac{1}{\left[1-\left(\frac{\xi}{8}\right)^{2} x^{2}\right]^{2}}\left(\left[1+\left(\frac{\xi}{8}\right)^{2} x^{2}\right] \delta_{m}^{a}-2\left(\frac{\xi}{8}\right)^{2} x^{a} x_{m}\right), \\
\omega_{m}^{a b} & =\frac{-2 \xi}{\left[1-\left(\frac{\xi}{8}\right)^{2} x^{2}\right]^{2}} \delta_{m}^{[a} x^{b]}=-\frac{8\left(\frac{\xi}{8}\right)}{\left(1-\left(\frac{\xi}{8}\right)^{2} x^{2}\right)^{2}}\left(x^{a} \delta_{m}^{b}-x^{b} \delta_{m}^{a}\right) .
\end{aligned}
$$


For completeness, let us also present the explicit form of the metric, the inverse vierbein and the inverse metric

$$
\begin{aligned}
g_{m n} & =\frac{1}{\left[1-\left(\frac{\xi}{8}\right)^{2} x^{2}\right]^{4}}\left(\left[1+\left(\frac{\xi}{8}\right)^{2} x^{2}\right]^{2} \eta_{m n}-4\left(\frac{\xi}{8}\right)^{2} x_{m} x_{n}\right), \\
e_{a}^{m} & =\frac{1-\left(\frac{\xi}{8}\right)^{2} x^{2}}{1+\left(\frac{\xi}{8}\right)^{2} x^{2}}\left(\left[1-\left(\frac{\xi}{8}\right)^{2} x^{2}\right] \delta_{a}^{m}+2\left(\frac{\xi}{8}\right)^{2} x_{a} x^{m}\right), \\
g^{m n} & =\frac{\left[1-\left(\frac{\xi}{8}\right)^{2} x^{2}\right]^{2}}{\left[1+\left(\frac{\xi}{8}\right)^{2} x^{2}\right]^{2}}\left(\left[1-\left(\frac{\xi}{8}\right)^{2} x^{2}\right]^{2} \eta^{m n}+4\left(\frac{\xi}{8}\right)^{2} x^{m} x^{n}\right) .
\end{aligned}
$$

It is well-known that the $\mathrm{AdS}_{D}$ metric (5.5) can be represented as an embedding in a flat $(D+1)$-dimensional space

$$
d s^{2}=\eta_{m n} d y^{m} d y^{n}-\left(d y^{D}\right)^{2},
$$

via the embedding constraint

$$
\eta_{m n} y^{m} y^{n}-\left(y^{D}\right)^{2}=-r^{2}
$$

Choosing the embedding coordinates for $\mathrm{AdS}_{4}$ to be

$$
y^{m}=\frac{1+\left(\frac{\xi}{8}\right)^{2} x^{2}}{\left[1-\left(\frac{\xi}{8}\right)^{2} x^{2}\right]^{2}} x^{m}, \quad y^{4}=\sqrt{r^{2}+x^{2} \frac{1+\left(\frac{\xi}{8}\right)^{2} x^{2}}{\left[1-\left(\frac{\xi}{8}\right)^{2} x^{2}\right]^{2}}},
$$

one readily recovers the metric (5.5), with the parameter $\xi$ being related to the $\mathrm{AdS}_{4}$ radius $r$ through

$$
\xi=\frac{2}{r}
$$

Finally, computing the Riemann tensor

$$
R_{m n}^{a b}=-32\left(\frac{\xi}{8}\right)^{2} \frac{1+\left(\frac{\xi}{8}\right)^{2} x^{2}}{\left[1-\left(\frac{\xi}{8}\right)^{2} x^{2}\right]^{4}}\left(\left[1+\left(\frac{\xi}{8}\right)^{2} x^{2}\right] \delta_{m}^{[a} \delta_{n}^{b]}+4\left(\frac{\xi}{8}\right)^{2} x^{[a} \delta_{[m}^{b]} x_{n]}\right),
$$

and the Ricci scalar

$$
R=-192\left(\frac{\xi}{8}\right)^{2}=-3 \xi^{2},
$$

one verifies that the metric (5.5) indeed corresponds to a space with constant negative curvature.

We are now in a position to consider $\operatorname{Sp}(2 n)$-invariant correlation functions of the hyperfields.

\section{Correlation functions on $\operatorname{Sp}(n)$ group manifold}

One can derive the generic form of $\operatorname{Sp}(2 n)$-invariant correlation functions for bosonic and fermionic fields of weight $-\frac{1}{2}$ on the $\operatorname{Sp}(n)$ group manifolds in a way similar to the conventional conformal field theories in various dimensions [45] (see also [46] for analogous 
computations in two-dimensional CFTs), as was carried out in [25] for computing the twoand three-point correlation functions in flat hyperspace. Because of the GL-flatness property of the hyperspaces, the correlation functions are related by the generalized conformal transformation.

For instance, since the two-point correlation functions for the flat-space fields $b$ and $f_{\mu}$ of conformal weight- $\frac{1}{2}$ satisfy the free equations, they are related to the corresponding two-point functions in $\operatorname{Sp}(n)$ in the same way as the fields themselves, i.e.

$$
\begin{aligned}
& \left\langle B\left(X_{1}\right) B\left(X_{2}\right)\right\rangle_{\operatorname{Sp}(n)}= \\
& c_{B}\left(\operatorname{det} G\left(X_{1}\right)\right)^{-\frac{1}{2}}\left(\operatorname{det} G\left(X_{2}\right)\right)^{-\frac{1}{2}}\left(\operatorname{det}\left|X_{12}\right|\right)^{-\frac{1}{2}} \\
& \left\langle F_{\alpha}\left(X_{1}\right) F_{\beta}\left(X_{2}\right)\right\rangle_{\operatorname{Sp}(n)}= \\
& c_{F} G_{\alpha}^{-1 \mu}\left(X_{1}\right) G_{\beta}^{-1 \nu}\left(X_{2}\right)\left(\operatorname{det} G\left(X_{1}\right)\right)^{-\frac{1}{2}}\left(\operatorname{det} G\left(X_{2}\right)\right)^{-\frac{1}{2}}\left(X_{12}\right)_{\mu \nu}^{-1}\left(\operatorname{det}\left|X_{12}\right|\right)^{-\frac{1}{2}},
\end{aligned}
$$

where $G\left(X_{1}\right)$ and $G\left(X_{2}\right)$ stand, respectively, for $G_{\alpha}{ }^{\beta}\left(X_{1}\right)$ and $G_{\alpha}{ }^{\beta}\left(X_{2}\right), c_{B}$ and $c_{F}$ are constants which are not fixed by the $\operatorname{Sp}(2 n)$ invariance, $X_{i j}=X_{i}-X_{j}$ and

$$
\begin{aligned}
\left\langle b\left(X_{1}\right) b\left(X_{2}\right)\right\rangle_{\text {flat }} & =c_{b}\left(\operatorname{det}\left|X_{12}\right|\right)^{-\frac{1}{2}}, \\
\left\langle f_{\mu}\left(X_{1}\right) f_{\nu}\left(X_{2}\right)\right\rangle_{\text {flat }} & =c_{f}\left(X_{12}\right)_{\mu \nu}^{-1}\left(\operatorname{det}\left|X_{12}\right|\right)^{-\frac{1}{2}}
\end{aligned}
$$

are the flat space correlation functions computed in [18].

Let us comment on the conformal dimensions of the various fields entering the correlation functions. For a flat hyperspace, the hyperfields have conformal weight $\Delta=\frac{1}{2}$ and are identified with the primary fields of the conformal field theory, whereas conformal fields with higher conformal weights correspond to derivatives of the hyperfields and are identified with the descendants. One may also consider primary fields of higher weight in hyperspace $[28,44]$ which are products of the master fields. For example, a bilinear combination of master fields corresponds to conserved currents and they are dual to master fields in higher dimensional hyperspaces. The detailed study of the generalized Conformal Field Theory involving such composite operators will be addressed in future work. Hence, in what follows, when relating correlation functions on flat and AdS hyperspaces, we will assume the fields to have weight $\frac{1}{2}$.

\subsection{Two-point functions}

Let us denote by

$$
\Phi\left(X_{1}, X_{2}\right)=\left\langle B\left(X_{1}\right) B\left(X_{2}\right)\right\rangle_{\mathrm{Sp}(n)},
$$

the two-point correlation function of two scalar fields of conformal weight $\frac{1}{2}$ on $\operatorname{Sp}(n)$. The invariance under the transformations (4.16) generated by the parameter $a^{\alpha \beta}$ results in the equation

$$
a^{\alpha \beta}\left(\mathcal{D}_{1, \alpha \beta}+\mathcal{D}_{2, \alpha \beta}\right) \Phi\left(X_{1}, X_{2}\right)=0
$$

In view of the identity

$$
\frac{\partial}{\partial X^{\alpha \beta}} \operatorname{det}|X|=X_{\alpha \beta}^{-1} \operatorname{det}|X|, \quad X^{\alpha \gamma} X_{\gamma \beta}^{-1}=\delta_{\beta}^{\alpha},
$$


equation (6.4) is solved by

$$
\Phi\left(X_{1}, X_{2}\right)=\left(\operatorname{det} G\left(X_{1}\right)\right)^{-\frac{1}{2}}\left(\operatorname{det} G\left(X_{2}\right)\right)^{-\frac{1}{2}} \tilde{\Phi}\left(\operatorname{det}\left|X_{12}\right|\right),
$$

where here, $\tilde{\Phi}\left(\operatorname{det}\left|X_{12}\right|\right)$ is an arbitrary function of $\operatorname{det}\left|X_{1}-X_{2}\right|$. Imposing also the invariance of the two-point function under the transformations generated by the parameter $g_{\alpha}{ }^{\beta}$, namely

$$
\left(g_{\alpha}{ }^{\alpha}+2 g_{\beta}{ }^{\alpha}\left(X_{1}^{\beta \gamma} \mathcal{D}_{1, \alpha \gamma}+X_{2}^{\beta \gamma} \mathcal{D}_{2, \alpha \gamma}\right)\right) \Phi\left(X_{1}, X_{2}\right)=0,
$$

fixes the form of the function $\tilde{\Phi}\left(\operatorname{det}\left|X_{1}-X_{2}\right|\right)$ and results in the following expression for the two-point function

$$
\Phi\left(X_{1}, X_{2}\right)=c_{B}\left(\operatorname{det} G\left(X_{1}\right)\right)^{-\frac{1}{2}}\left(\operatorname{det} G\left(X_{2}\right)\right)^{-\frac{1}{2}}\left(\operatorname{det}\left|X_{12}\right|\right)^{-\frac{1}{2}},
$$

where $c_{B}$ is an arbitrary constant. Finally, the invariance under the transformations generated by the parameters $k^{\alpha \beta}$ imposes the condition

$$
k_{\alpha \beta}\left(\frac{1}{2} X_{1}^{\alpha \beta}+X_{1}^{\alpha \gamma} X_{1}^{\beta \delta} \mathcal{D}_{1, \gamma \delta}+\frac{1}{2} X_{2}^{\alpha \beta}+X_{2}^{\alpha \gamma} X_{2}^{\beta \delta} \mathcal{D}_{2, \gamma \delta}\right) \Phi\left(X_{1}, X_{2}\right)=0,
$$

which is identically satisfied by (6.8).

The derivation of eq. (6.8) reproduces the relation (6.1) between the $\operatorname{Sp}(n)$ two-point functions of scalar fields of conformal weight $\frac{1}{2}$ with those in flat hyperspace computed in [25]. Analogously, one may check the relation (6.2) between the two-point functions of two spinor fields of weight $\frac{1}{2}$.

\subsection{Three-point functions}

The calculation of the weight- $\frac{1}{2}$ bosonic field three-point function

$$
\Phi\left(X_{1}, X_{2}, X_{3}\right)=\left\langle B\left(X_{1}\right) B\left(X_{2}\right) B\left(X_{3}\right)\right\rangle_{\mathrm{Sp}(n)},
$$

proceeds in a similar way. From the equation

$$
a^{\alpha \beta}\left(\mathcal{D}_{1, \alpha \beta}+\mathcal{D}_{2, \alpha \beta}+\mathcal{D}_{3, \alpha \beta}\right) \Phi\left(X_{1}, X_{2}, X_{3}\right)=0,
$$

one obtains

$$
\begin{aligned}
\Phi\left(X_{1}, X_{2}, X_{3}\right)= & c_{3}\left(\operatorname{det} G\left(X_{1}\right)\right)^{-\frac{1}{2}}\left(\operatorname{det} G\left(X_{2}\right)\right)^{-\frac{1}{2}}\left(\operatorname{det} G\left(X_{3}\right)\right)^{-\frac{1}{2}} \\
& \tilde{\Phi}\left(\operatorname{det}\left|X_{12}\right|, \operatorname{det}\left|X_{23}\right|, \operatorname{det}\left|X_{13}\right|\right),
\end{aligned}
$$

where $\tilde{\Phi}$ is an arbitrary function depending only on the combinations $\operatorname{det}\left|X_{i}-X_{j}\right|$. The equation

$$
\left(\frac{3}{2} g_{\alpha}{ }^{\alpha}+2 g_{\beta}{ }^{\alpha} \sum_{i=1}^{i=3} X_{i}^{\beta \gamma} \mathcal{D}_{i, \alpha \gamma}\right) F\left(X_{1}, X_{2}, X_{3}\right)=0,
$$

then fixes the form of the function $\Phi$ to be

$$
\begin{aligned}
\Phi\left(X_{1}, X_{2}, X_{3}\right)= & \left(\operatorname{det} G\left(X_{1}\right)\right)^{-\frac{1}{2}}\left(\operatorname{det} G\left(X_{2}\right)\right)^{-\frac{1}{2}}\left(\operatorname{det} G\left(X_{3}\right)\right)^{-\frac{1}{2}} \\
& \left(\operatorname{det}\left|X_{12}\right|\right)^{-\frac{k_{3}}{2}}\left(\operatorname{det}\left|X_{23}\right|\right)^{-\frac{k_{1}}{2}}\left(\operatorname{det}\left|X_{13}\right|\right)^{-\frac{k_{2}}{2}},
\end{aligned}
$$


with

$$
k_{1}+k_{2}+k_{3}=\frac{3}{2}
$$

Finally, the equation

$$
k_{\alpha \beta} \sum_{i=1}^{i=3}\left(\frac{1}{2} X_{i}^{\alpha \beta}+X_{i}^{\alpha \gamma} X_{i}^{\beta \delta} \mathcal{D}_{i, \gamma \delta}\right) \Phi\left(X_{1}, X_{2}, X_{3}\right)=0,
$$

implies that

$$
k_{1}=k_{2}=k_{3}=\frac{1}{2} .
$$

The equation (6.14) relates (via the conformal factors $\left(\operatorname{det} G\left(X_{1}\right)\right)^{-\frac{1}{2}}\left(\operatorname{det} G\left(X_{2}\right)\right)^{-\frac{1}{2}}$ $\left.\left(\operatorname{det} G\left(X_{3}\right)\right)^{-\frac{1}{2}}\right)$ the bosonic field three-point function on $\operatorname{Sp}(n)$ with that in flat hyperspace computed in [25]. In a similar way, one observes that the three-point function involving two spinor and one scalar fields ${ }^{6}$ are related as follows

$$
\begin{aligned}
& \left\langle F_{\alpha}\left(X_{1}\right) F_{\beta}\left(X_{2}\right) B\left(X_{3}\right)\right\rangle_{\operatorname{Sp}(n)}= \\
& \quad c_{3 f}\left(\operatorname{det} G\left(X_{1}\right)\right)^{-\frac{1}{2}}\left(\operatorname{det} G\left(X_{2}\right)\right)^{-\frac{1}{2}}\left(\operatorname{det} G\left(X_{3}\right)\right)^{-\frac{1}{2}} \\
& \quad G_{\alpha}^{-1 \mu}\left(X_{1}\right) G_{\beta}^{-1 \nu}\left(X_{2}\right)\left(X_{12}\right)_{\mu \nu}^{-1}\left(\operatorname{det}\left|X_{12}\right|\right)^{-\frac{1}{4}}\left(\operatorname{det} X_{23} \mid\right)^{-\frac{1}{4}}\left(\operatorname{det}\left|X_{13}\right|\right)^{-\frac{1}{4}} .
\end{aligned}
$$

\subsection{Four-point functions}

To the best of our knowledge, the explicit form of $N$-point functions for $N \geq 4$, both in flat and $\operatorname{Sp}(n)$ hyperspace, have not previously been given in the literature, and below we present the result for the four-point correlation functions. ${ }^{7}$

The computation of the $\mathrm{Sp}(2 n)$-invariant four-point functions follows the same lines as the computation of the two- and three-point functions. Let us consider first the correlation function of four scalar fields of an arbitrary weight $\Delta$ in flat hyperspace. Its invariance under the translations

$$
a^{\alpha \beta} \sum_{i=1}^{4} \frac{\partial}{\partial X_{i}^{\alpha \beta}} \Phi\left(X_{1}, X_{2}, X_{3}, X_{4}\right)=0,
$$

implies that the function $\Phi\left(X_{1}, X_{2}, X_{3}, X_{4}\right)$ depends only on the differences $X_{i j}=X_{i}-X_{j}$. Using the analogy with the usual conformal field theory for a four-point function we write

$$
\Phi\left(X_{1}, X_{2}, X_{3}, X_{4}\right)=c_{4} \prod_{i j, i<j} \frac{1}{\left(\operatorname{det}\left|X_{i j}\right|\right)^{\Gamma_{i j}}} \tilde{\Phi}\left(z, z^{\prime}\right),
$$

where $z, z^{\prime}$ are the two independent cross-ratios

$$
z=\operatorname{det}\left(\frac{\left|X_{12}\right|\left|X_{34}\right|}{\left|X_{13}\right|\left|X_{24}\right|}\right), z^{\prime}=\operatorname{det}\left(\frac{\left|X_{12}\right|\left|X_{34}\right|}{\left|X_{23}\right|\left|X_{14}\right|}\right)
$$

\footnotetext{
${ }^{6}$ The correlation functions containing an odd number of spinor fields vanish identically [25].

${ }^{7}$ In the unfolded formulation of hyperspace dynamics, $N$-point correlation functions were computed in [26], however their relation to our form of the correlators still remains to be understood.
} 
Crossing symmetry then implies the constraint

$$
\tilde{\Phi}\left(z, z^{\prime}\right)=\tilde{\Phi}\left(\frac{1}{z}, \frac{z^{\prime}}{z}\right)=\tilde{\Phi}\left(\frac{z}{z^{\prime}}, \frac{1}{z^{\prime}}\right) .
$$

Then, requiring the invariance under the GL $(n)$ transformations, namely

$$
\left(g_{\alpha}{ }^{\alpha} \sum_{i=1}^{i=4} \Delta_{i}+2 g_{\beta}{ }^{\alpha} \sum_{i=1}^{i=4} X_{i}^{\beta \gamma} \frac{\partial}{\partial X_{i}^{\alpha \beta}}\right) \Phi\left(X_{1}, X_{2}, X_{3}, X_{4}\right)=0,
$$

one obtains the additional condition

$$
\sum_{j=2}^{j=4} \sum_{i=1}^{i=j-1} \Gamma_{i j}=\frac{1}{2} \sum_{i=1}^{i=4} \Delta_{i}
$$

Finally, the invariance under the generalized conformal boosts

$$
k_{\alpha \beta} \sum_{i=1}^{i=4}\left(\Delta_{i} X_{i}^{\alpha \beta}+X_{i}^{\alpha \gamma} X_{i}^{\beta \delta} \frac{\partial}{\partial X_{i}^{\gamma \delta}}\right) \Phi\left(X_{1}, X_{2}, X_{3}, X_{4}\right)=0,
$$

imposes the condition on the conformal weights

$$
\sum_{j} \Gamma_{i j}=\Delta_{i}, \quad i \neq j
$$

Let us note that, similar to the usual conformal field theory, the four-point function (6.20) contains an arbitrary function $\tilde{\Phi}$, whose argument can be considered as a generalization of the cross-ratios to the case of matrix-valued coordinates $X^{\mu \nu}$.

Now, as in the case of the two- and three-point functions, the expressions for four-point functions on the $\operatorname{Sp}(n)$ group manifold for the primary weight- $\frac{1}{2}$ fields can be obtained from the corresponding expressions on the flat hyperspace by the re-scaling of the former with appropriate factors of $\left(\operatorname{det} G\left(X_{i}\right)\right)^{-\frac{1}{2}}$, i.e.

$$
\Phi\left(X_{1}, X_{2}, X_{3}, X_{4}\right)_{\operatorname{Sp}(4)}=\Phi\left(X_{1}, X_{2}, X_{3}, X_{4}\right) \prod_{i=1}^{4}\left(\operatorname{det} G\left(X_{i}\right)\right)^{-\frac{1}{2}},
$$

where the function $\Phi\left(X_{1}, X_{2}, X_{3}, X_{4}\right)$ on the right hand side of this equation is given in (6.20).

In the same way, one can obtain the four-point function of four spinor fields of weight $\Delta=\frac{1}{2}$ by rescaling the flat hyperspace result

$$
\begin{aligned}
& \left\langle F_{\alpha}\left(X_{1}\right) F_{\beta}\left(X_{2}\right) F_{\gamma}\left(X_{3}\right) F_{\delta}\left(X_{4}\right)\right\rangle_{\mathrm{Sp}(4)}= \\
& \quad G_{\alpha}^{-1^{\mu}}\left(X_{1}\right) G_{\beta}^{-1^{\nu}}\left(X_{2}\right) G_{\gamma}^{-1 \rho}\left(X_{3}\right) G_{\delta}^{-1^{\sigma}}\left(X_{4}\right) \\
& \quad \times \prod_{i=1}^{4}\left(\operatorname{det} G\left(X_{i}\right)\right)^{-\frac{1}{2}}\left\langle F_{\mu}\left(X_{1}\right) F_{\nu}\left(X_{2}\right) F_{\rho}\left(X_{3}\right) F_{\sigma}\left(X_{4}\right)\right\rangle_{\text {flat }} \\
& \left\langle F_{\mu}\left(X_{1}\right) F_{\nu}\left(X_{2}\right) F_{\rho}\left(X_{3}\right) F_{\sigma}\left(X_{4}\right)\right\rangle_{\text {flat }}= \\
& \quad \prod_{i<j} \operatorname{det}\left|X_{i j}\right|^{-\frac{1}{3}}\left[\left(X_{12}\right)_{\mu \nu}^{-1}\left(X_{34}\right)_{\rho \sigma}^{-1} \Phi_{12,34}\left(z, z^{\prime}\right)\right. \\
& \left.\quad-\left(X_{13}\right)_{\mu \rho}^{-1}\left(X_{24}\right)_{\nu \sigma}^{-1} \Phi_{13,24}\left(z, z^{\prime}\right)+\left(X_{14}\right)_{\mu \sigma}^{-1}\left(X_{23}\right)_{\nu \rho}^{-1} \Phi_{14,23}\left(z, z^{\prime}\right)\right] .
\end{aligned}
$$


As before, the functions $\Phi_{i j, k \ell}\left(z, z^{\prime}\right)$ are indeterminate functions of the crossing ratios constrained by crossing symmetry to satisfy

$$
\Phi_{12,34}\left(z, z^{\prime}\right)=\Phi_{13,24}\left(\frac{1}{z}, \frac{z^{\prime}}{z}\right)=\Phi_{14,23}\left(\frac{z}{z^{\prime}}, \frac{1}{z^{\prime}}\right) .
$$

Finally, one may obtain the four-point function that involves two spinorial and two bosonic fields on flat hyperspace

$$
\left\langle F_{\mu}\left(X_{1}\right) F_{\nu}\left(X_{2}\right) b\left(X_{3}\right) b\left(X_{4}\right)\right\rangle_{\text {flat }}=\left(X_{12}\right)_{\mu \nu}^{-1} \tilde{\Psi}\left(z, z^{\prime}\right) \prod_{i<j} \operatorname{det}\left|X_{i j}\right|^{-\frac{1}{3}},
$$

and relate it to the corresponding correlator on $\operatorname{Sp}(n)$

$$
\begin{aligned}
& \left\langle F_{\alpha}\left(X_{1}\right) F_{\beta}\left(X_{2}\right) b\left(X_{3}\right) b\left(X_{4}\right)\right\rangle_{\mathrm{Sp}(4)}= \\
& \quad G_{\alpha}^{-1^{\mu}}\left(X_{1}\right) G_{\beta}^{-1^{\nu}}\left(X_{2}\right) \prod_{i=1}^{4}\left(\operatorname{det} G\left(X_{i}\right)\right)^{-\frac{1}{2}}\left\langle F_{\mu}\left(X_{1}\right) F_{\nu}\left(X_{2}\right) b\left(X_{3}\right) b\left(X_{4}\right)\right\rangle_{\mathrm{flat}} .
\end{aligned}
$$

In eq. (6.31), again, $\Psi\left(z, z^{\prime}\right)$ is a function satisfying the crossing relations (6.22) that cannot be determined by the $\operatorname{Sp}(2 n)$-symmetry alone. In fact, the functions of the cross-ratios $\tilde{\Phi}\left(z, z^{\prime}\right), \Phi_{i k, k \ell}\left(z, z^{\prime}\right)$ and $\Psi\left(z, z^{\prime}\right)$ should be completely determined in terms of the OPE between primary fields in the CFT on flat hyperspace.

\section{Conclusion}

In this paper we have considered some aspects of the $\operatorname{Sp}(2 n)$ invariant formulation of higher spin fields. The main advantage of this approach is that one may combine infinite series of higher spin fields into one scalar and one spinor "master" field defined on a hyperspace. It is then possible to study their field equations, correlation functions and other properties by making appropriate generalizations of analogous quantities for scalar and spinor fields on conventional flat and AdS spaces.

We have considered the theory on both, the flat hyperspace and the $\operatorname{Sp}(n)$ group manifold, the latter being a hyperspace extension of $\mathrm{AdS}_{d}$ space. As we mentioned above, it is quite instructive to follow the analogy between the properties of conformal scalar and spinor fields on a flat space and a scalar and a spinor field on anti de Sitter space on the one side, and a scalar and spinor field on flat and $\operatorname{Sp}(n)$ hyperspace on the other side.

Typically, computations on the ordinary AdS space are performed in a particular conformally flat parametrization of the metric. Similarly, our study of the field equations and correlation functions on $\operatorname{Sp}(n)$ group manifolds has been heavily based on the $\operatorname{GL}(n)$ flatness property of these manifolds. By exploiting this property, we have established, via the generalized conformal transformation, the relation between the field equations for scalar and spinor "master" fields on flat and $\operatorname{Sp}(n)$ group manifolds.

Provided that the fields have an appropriated conformal weight $\left(\Delta=\frac{1}{2}\right)$, their equations of motion on flat hyperspace and $\operatorname{Sp}(n)$ group manifold are invariant under the $\operatorname{Sp}(2 n)$ symmetry, which is a generalization of the usual conformal symmetry to the case of the 
hyperspaces. Using a technique similar to that of multidimensional conformal field theories [45], we have extended the results of $[18,25]$ on two- and three-point functions of the scalar and spinor hyperfields on flat hyperspace by deriving the form of the two- and three-point correlation functions on $\operatorname{Sp}(n)$ and four-point functions on flat and $\operatorname{Sp}(n)$ hyperspace exploiting their invariance under the $\operatorname{Sp}(2 n)$ group. The correlation functions on the $\operatorname{Sp}(n)$ manifolds are related to their flat hyperspace counterparts by the the generalized conformal transformation similar to that related the fields themselves.

Let us note that the results obtained in this paper are valid for $\operatorname{Sp}(2 n)$ invariant theories with an arbitrary value of $n$. By now, however, the most studied physically interesting example has been the case of $n=4$ which corresponds to the four-dimensional higher spin gauge theory. In this case, the master fields $b(X)$ and $f_{\alpha}(X)$ contain the curvatures of all the higher spin fields (with the spin ranging from zero to infinity) and the hyperfield equations of motion encode the Bianchi identities and the field equations for the higher spin curvatures explicitly derived in the flat space case only (see section 2). So it will be instructive to derive in a similar way the Bianchi identities and the equations of motion for the higher spin curvatures in $\mathrm{AdS}_{4}$ from the field equations (3.14)-(3.17) on $\mathrm{Sp}(4)$. In this case it is not consistent anymore to naively extend the $\operatorname{Sp}(4)$ hyperfields $B(x, y)$ and $F_{\alpha}(x, y)$ as series in the powers of the tensorial coordinates $y^{m n}$ (as in the flat case (2.14)), but one should rather perform the expansion in the Lorentz harmonics of the group $\mathrm{SO}(3,1) \subset \mathrm{Sp}(4)$ parametrized by $y^{m n}$, very much like when performing the series expansion of fields in Kaluza-Klein theories. To this end, it might be useful to choose a different parametrization of the $\mathrm{Sp}(4)$ group manifold of the form $\mathcal{O}\left(x^{l}, y^{m n}\right)=K(x) H(y)$, where $K(x)$ is the $\mathrm{AdS}_{4}$ coset element and $H(y)$ is the $\mathrm{SO}(3,1)$ group element.

It would also be of interest to apply the results of this paper to the description of higher spin theories in higher dimensions, especially in AdS. For instance, the cases of $n=8$ and $n=16$ correspond, respectively, to conformal higher spin fields on six- and tendimensional space-time, which was demonstrated for theories in flat (hyper)space in [24], while the extension of these results to theories in AdS is still to be carried out.

We hope that the results obtained in this paper will be useful for better understanding generic conformal properties of higher spin fields (see e.g. [47-53]), as well as for further study of higher spin AdS/CFT duality.

However, the most ambitious issue in this kind of theories is the interaction problem. As we have seen, the generalized conformal field theories in hyperspaces considered above have nontrivial three- and four-point correlation functions, however this does not yet imply the existence of non-trivial interactions. One should resort to additional criteria, such as the study of anomalous dimensions of conformal operators etc. See e.g. [54] for the discussion of these issues in conventional CFTs. If non-trivial interactions of scalar and spinor hyperfields exist, from the perspective of higher spin field theory they should correspond to higher-order terms in higher spin curvatures contained inside the hyperfields. This is yet another important open problem of how to incorporate higher spin potentials directly into the hyperspace framework. So far this has only been done with the use of the unfolded technique [15].

So, it seems to be particularly interesting to perform a further detailed study of properties of conformal field theories on flat hyperspaces and $\operatorname{Sp}(n)$ group manifolds and, in par- 
ticular, the possibility of constructing interacting conformal field theories on these spaces. In this respect, let us note that upon having verified the consistency associated with gauge invariance etc. (see e.g. [11]-[14, 55] and references therein), consistency checks of interacting higher spin gauge theories on flat and AdS backgrounds proceed in different ways. For a flat background extra crucial constraints on consistent interactions are obtained by requiring the existence of a nontrivial S-matrix (in contrast to the theories on AdS backgrounds in which the conventional notion of S-matrix is not applicable) and these constraints appear when one considers quartic interaction vertices [56-59].

\section{Acknowledgments}

We are grateful to P. Dempster, S. Kuzenko, A. Petkou, I. Samsonov, E. Skvortsov and M. Vasiliev for fruitful discussions. I.F. would like to acknowledge the Theory Division at CERN and the Max-Planck-Institut für Gravitationsphysik in Potsdam (Golm) for their warm hospitality during the later stages of this work. Work of D.S. was partially supported by the MIUR-PRIN contract 2009-KHZKRX and by the INFN Special Initiative TV12. D.S. acknowledges hospitality and support extended to him at CBPF, Rio de Janeiro and at ICTP SAIFR, Sao Paulo, Brazil during the Workshop "Higher-Spin and Higher-Curvature Gravity" at the final stage of this project. M.T. would like to thank the Department of Physics, the University of Auckland, where part of this work has been performed, for its kind hospitality. Work of M.T. has been supported in part by an Australian Research Council grant DP120101340. M.T. would also like to acknowledge grant 31/89 of the Rustaveli National Science Foundation.

\section{A Proof of the GL $(n)$ flatness, some technical details and useful identi- ties}

We use the following normalization for the Dirac $\gamma$-matrices

$$
\left(\gamma^{m}\right)^{\alpha}{ }_{\delta}\left(\gamma^{n}\right)^{\delta}{ }_{\beta}+\left(\gamma^{n}\right)^{\alpha}{ }_{\delta}\left(\gamma^{m}\right)^{\delta}{ }_{\beta}=2 \eta^{m n} \delta_{\beta}^{\alpha},
$$

where $m, n$ and other Latin letters are space-time vector indices, and $\alpha, \beta$ and other Greek letters correspond to spinorial indices. Throughout the paper "(, )" denotes symmetrization and "[, ]" denotes antisymmetrization with weight one. The symplectic matrix $C^{\alpha \beta}=-C^{\beta \alpha}$ is used to relate upper and lower spinorial indexes as follows

$$
\mu^{\alpha}=C^{\alpha \beta} \mu_{\beta}, \quad \mu_{\alpha}=-C_{\alpha \beta} \mu^{\beta}, \quad C^{\alpha \gamma} C_{\gamma \beta}=-\delta_{\beta}^{\alpha} .
$$

We shall now show that the Cartan form given by (4.3)-(4.4) indeed solves the MaurerCartan equation (3.4). Consider first the $d \Omega^{\alpha \beta}$ term in the Maurer-Cartan equation

$$
\begin{aligned}
d\left(d X^{\alpha^{\prime} \beta^{\prime}} G_{\alpha^{\prime}}{ }^{\alpha} G_{\beta^{\prime}}{ }^{\beta}\right)= & d X^{\alpha^{\prime} \beta^{\prime}} d X^{\gamma \delta}\left(\left(\frac{\partial G_{\alpha^{\prime}}{ }^{\alpha}}{\partial X^{\gamma \delta}}\right) G_{\beta^{\prime}}{ }^{\beta}+G_{\alpha^{\prime}}{ }^{\alpha}\left(\frac{\partial G_{\beta^{\prime}}{ }^{\beta}}{\partial X^{\gamma \delta}}\right)\right) \\
= & -d X^{\alpha^{\prime} \beta^{\prime}} d X^{\gamma \delta} G_{\alpha^{\prime}}{ }^{\sigma} \frac{\partial G_{\sigma}^{-1 \rho}}{\partial X^{\gamma \delta}} G_{\rho}{ }^{\alpha} G_{\beta^{\prime}}{ }^{\beta} \\
& -d X^{\alpha^{\prime} \beta^{\prime}} d X^{\gamma \delta} G_{\alpha^{\prime}}{ }^{\alpha} G_{\beta^{\prime}} \frac{\partial G_{\sigma}^{-1 \rho}}{\partial X^{\gamma \delta}} G_{\rho}{ }^{\beta} .
\end{aligned}
$$


Using

$$
\frac{d X^{\alpha \beta}}{d X^{\gamma \delta}}=\frac{1}{2}\left(\delta_{\gamma}^{\alpha} \delta_{\delta}^{\beta}+\delta_{\gamma}^{\beta} \delta_{\delta}^{\alpha}\right)
$$

and the explicit form of the inverse matrix (4.2), one obtains

$$
\frac{\xi}{4}\left(d X^{\beta^{\prime} \sigma} G_{\sigma \rho} d X^{\rho \alpha^{\prime}}+d X^{\alpha^{\prime} \sigma} G_{\sigma \rho} d X^{\rho \beta^{\prime}}\right) G_{\alpha^{\prime}}{ }^{\alpha} G_{\beta^{\prime}}{ }^{\beta} .
$$

Let us note, that the product of an even number of $X^{\alpha \beta}$ matrices is antisymmetric in spinorial indexes, whereas the product of an odd number of $X^{\alpha \beta}$ is a symmetric matrix. For example,

$$
X^{\alpha \gamma} X_{\gamma}{ }^{\beta}=-X^{\beta \gamma} X_{\gamma}{ }^{\alpha}, \quad X^{\alpha}{ }_{\gamma} X_{\delta}^{\gamma} X^{\delta \beta}=+X_{\delta}^{\beta} X_{\gamma}^{\delta} X^{\gamma \alpha}, \quad \text { etc. }
$$

As a result, equation (A.4) reduces to

$$
\left.\frac{\xi}{2} d X^{\beta^{\prime} \sigma} G_{\sigma \rho}\right|_{\mathrm{even}} d X^{\rho \alpha^{\prime}} G_{\alpha^{\prime}}{ }^{\alpha} G_{\beta^{\prime}}{ }^{\beta}=\frac{\xi}{2} d X^{\beta^{\prime} \sigma} G_{[\sigma \rho]} d X^{\rho \alpha^{\prime}} G_{\alpha^{\prime}}{ }^{\alpha} G_{\beta^{\prime}}{ }^{\beta} .
$$

The evaluation of the second term in the Maurer-Cartan equation (3.4) is straightforward with the use of (4.11). After doing so, it is easy to see that the Cartan form (4.3)-(4.4) solves the Maurer-Cartan equation (3.4).

Open Access. This article is distributed under the terms of the Creative Commons Attribution License (CC-BY 4.0), which permits any use, distribution and reproduction in any medium, provided the original author(s) and source are credited.

\section{References}

[1] C. Fronsdal, Massless fields with integer spin, Phys. Rev. D 18 (1978) 3624 [INSPIRE].

[2] M.A. Vasiliev, 'Gauge' form of description of massless fields with arbitrary spin (in Russian), Yad. Fiz. 32 (1980) 855 [INSPIRE].

[3] C. Aragone and S. Deser, Higher spin vierbein gauge fermions and hypergravities, Nucl. Phys. B 170 (1980) 329 [InSPIRE].

[4] M.A. Vasiliev, Consistent equation for interacting gauge fields of all spins in (3+1)-dimensions, Phys. Lett. B 243 (1990) 378 [InSPIRE].

[5] M.A. Vasiliev, More on equations of motion for interacting massless fields of all spins in (3+1)-dimensions, Phys. Lett. B 285 (1992) 225 [INSPIRE].

[6] M.A. Vasiliev, Nonlinear equations for symmetric massless higher spin fields in (A) $d S_{d}$, Phys. Lett. B 567 (2003) 139 [hep-th/0304049] [INSPIRE].

[7] M.A. Vasiliev, Higher spin gauge theories: star product and AdS space, hep-th/9910096 [INSPIRE].

[8] D. Sorokin, Introduction to the classical theory of higher spins, AIP Conf. Proc. 767 (2005) 172 [hep-th/0405069] [INSPIRE].

[9] X. Bekaert, S. Cnockaert, C. Iazeolla and M.A. Vasiliev, Nonlinear higher spin theories in various dimensions, hep-th/0503128 [INSPIRE]. 
[10] N. Bouatta, G. Compere and A. Sagnotti, An introduction to free higher-spin fields, hep-th/0409068 [INSPIRE].

[11] A. Fotopoulos and M. Tsulaia, Gauge invariant Lagrangians for free and interacting higher spin fields. A review of the BRST formulation, Int. J. Mod. Phys. A 24 (2009) 1 [arXiv: 0805.1346] [INSPIRE].

[12] X. Bekaert, N. Boulanger and P. Sundell, How higher-spin gravity surpasses the spin two barrier: no-go theorems versus yes-go examples, Rev. Mod. Phys. 84 (2012) 987 [arXiv: 1007.0435] [INSPIRE].

[13] A. Sagnotti, Notes on strings and higher spins, J. Phys. A 46 (2013) 214006 [arXiv:1112.4285] [INSPIRE].

[14] M. Tsulaia, On tensorial spaces and BCFW recursion relations for higher spin fields, Int. J. Mod. Phys. A 27 (2012) 1230011 [arXiv:1202.6309] [INSPIRE].

[15] M.A. Vasiliev, On conformal, $\mathrm{SL}(4, R)$ and $\operatorname{Sp}(8, R)$ symmetries of $4 d$ massless fields, Nucl. Phys. B 793 (2008) 469 [arXiv:0707.1085] [INSPIRE].

[16] I.A. Bandos and J. Lukierski, Tensorial central charges and new superparticle models with fundamental spinor coordinates, Mod. Phys. Lett. A 14 (1999) 1257 [hep-th/9811022] [INSPIRE].

[17] I.A. Bandos, J. Lukierski and D.P. Sorokin, Superparticle models with tensorial central charges, Phys. Rev. D 61 (2000) 045002 [hep-th/9904109] [INSPIRE].

[18] M.A. Vasiliev, Relativity, causality, locality, quantization and duality in the $\operatorname{Sp}(2 M)$ invariant generalized space-time, hep-th/0111119 [INSPIRE].

[19] C. Fronsdal, Massless particles, orthosymplectic symmetry and another type of Kaluza-Klein theory, UCLA-85-TEP-10, in Essays on supersymmetry, Mathematical Physics Studies, Reidel, Dordrecht Netherlands (1986), pp. 163-265 [INSPIRE].

[20] M. Flato and C. Fronsdal, One massless particle equals two Dirac singletons: elementary particles in a curved space. VI, Lett. Math. Phys. 2 (1978) 421 [INSPIRE].

[21] M.A. Vasiliev, Conformal higher spin symmetries of $4 D$ massless supermultiplets and $\operatorname{osp}(L, 2 M)$ invariant equations in generalized (super)space, Phys. Rev. D 66 (2002) 066006 [hep-th/0106149] [INSPIRE].

[22] M. Plyushchay, D. Sorokin and M. Tsulaia, Higher spins from tensorial charges and $\operatorname{OSp}(N \mid 2 n)$ symmetry, JHEP 04 (2003) 013 [hep-th/0301067] [INSPIRE].

[23] M. Plyushchay, D. Sorokin and M. Tsulaia, GL flatness of $\mathrm{OSp}(1 \mid 2 n)$ and higher spin field theory from dynamics in tensorial spaces, hep-th/0310297 [INSPIRE].

[24] I. Bandos, X. Bekaert, J.A. de Azcarraga, D. Sorokin and M. Tsulaia, Dynamics of higher spin fields and tensorial space, JHEP 05 (2005) 031 [hep-th/0501113] [INSPIRE].

[25] M.A. Vasiliev and V.N. Zaikin, On $\operatorname{Sp}(2 M)$ invariant Green functions, Phys. Lett. B 587 (2004) 225 [hep-th/0312244] [INSPIRE].

[26] V.E. Didenko and E.D. Skvortsov, Exact higher-spin symmetry in CFT: all correlators in unbroken Vasiliev theory, JHEP 04 (2013) 158 [arXiv:1210.7963] [INSPIRE].

[27] N. Colombo and P. Sundell, Higher spin gravity amplitudes from zero-form charges, arXiv: 1208.3880 [INSPIRE]. 
[28] O.A. Gelfond and M.A. Vasiliev, Higher rank conformal fields in the $\operatorname{Sp}(2 M)$ symmetric generalized space-time, Theor. Math. Phys. 145 (2005) 1400 [hep-th/0304020] [INSPIRE].

[29] I. Bandos, P. Pasti, D. Sorokin and M. Tonin, Superfield theories in tensorial superspaces and the dynamics of higher spin fields, JHEP 11 (2004) 023 [hep-th/0407180] [INSPIRE].

[30] E. Ivanov, Nonlinear realizations in tensorial superspaces and higher spins, hep-th/0703056 [INSPIRE].

[31] O.A. Gelfond and M.A. Vasiliev, Higher spin fields in Siegel space, currents and theta functions, JHEP 03 (2009) 125 [arXiv:0801.2191] [INSPIRE].

[32] O.A. Gelfond and M.A. Vasiliev, $\mathrm{Sp}(8)$ invariant higher spin theory, twistors and geometric BRST formulation of unfolded field equations, JHEP 12 (2009) 021 [arXiv:0901.2176] [INSPIRE].

[33] O.A. Gelfond and M.A. Vasiliev, Unfolded equations for current interactions of $4 d$ massless fields as a free system in mixed dimensions, arXiv:1012.3143 [INSPIRE].

[34] I.A. Bandos, J.A. de Azcarraga and C. Meliveo, Extended supersymmetry in massless conformal higher spin theory, Nucl. Phys. B 853 (2011) 760 [arXiv:1106.5199] [InSPIRE].

[35] S. Fedoruk and J. Lukierski, New spinorial particle model in tensorial space-time and interacting higher spin fields, JHEP 02 (2013) 128 [arXiv:1210.1506] [INSPIRE].

[36] I.A. Bandos, J. Lukierski, C. Preitschopf and D.P. Sorokin, OSp supergroup manifolds, superparticles and supertwistors, Phys. Rev. D 61 (2000) 065009 [hep-th/9907113] [INSPIRE].

[37] I.A. Bandos, J. Lukierski and D.P. Sorokin, The OSp(1|4) superparticle and exotic BPS states, hep-th/9912264 [INSPIRE].

[38] V.E. Didenko and M.A. Vasiliev, Free field dynamics in the generalized AdS (super)space, J. Math. Phys. 45 (2004) 197 [hep-th/0301054] [INSPIRE].

[39] S. Weinberg, Photons and gravitons in perturbation theory: derivation of Maxwell's and Einstein's equations, Phys. Rev. 138 (1965) B988 [INSPIRE].

[40] B. de Wit and D.Z. Freedman, Systematics of higher spin gauge fields, Phys. Rev. D 21 (1980) 358 [InSPIRE].

[41] P.S. Howe, S. Penati, M. Pernici and P.K. Townsend, Wave equations for arbitrary spin from quantization of the extended supersymmetric spinning particle, Phys. Lett. B 215 (1988) 555 [INSPIRE].

[42] X. Bekaert and N. Boulanger, Tensor gauge fields in arbitrary representations of $\operatorname{GL}(D, R)$ : duality and Poincaré lemma, Commun. Math. Phys. 245 (2004) 27 [hep-th/0208058] [INSPIRE].

[43] X. Bekaert and N. Boulanger, On geometric equations and duality for free higher spins, Phys. Lett. B 561 (2003) 183 [hep-th/0301243] [INSPIRE].

[44] O.A. Gelfond and M.A. Vasiliev, Higher-rank fields and currents, arXiv:1312.6673 [INSPIRE].

[45] H. Osborn and A.C. Petkou, Implications of conformal invariance in field theories for general dimensions, Annals Phys. 231 (1994) 311 [hep-th/9307010] [INSPIRE].

[46] A.M. Polyakov, Conformal symmetry of critical fluctuations, JETP Lett. 12 (1970) 381 [INSPIRE]. 
[47] R.R. Metsaev, CFT adapted gauge invariant formulation of arbitrary spin fields in AdS and modified de Donder gauge, Phys. Lett. B 671 (2009) 128 [arXiv:0808.3945] [INSPIRE].

[48] R.R. Metsaev, Gauge invariant two-point vertices of shadow fields, AdS/CFT and conformal fields, Phys. Rev. D 81 (2010) 106002 [arXiv:0907.4678] [INSPIRE].

[49] R.R. Metsaev, CFT adapted approach to massless fermionic fields, AdS/CFT and fermionic conformal fields, arXiv:1311.7350 [INSPIRE].

[50] A.R. Gover, A. Shaukat and A. Waldron, Tractors, mass and Weyl invariance, Nucl. Phys. B 812 (2009) 424 [arXiv:0810.2867] [InSPIRE].

[51] M.S. Costa, J. Penedones, D. Poland and S. Rychkov, Spinning conformal correlators, JHEP 11 (2011) 071 [arXiv: 1107.3554] [INSPIRE].

[52] J. Maldacena and A. Zhiboedov, Constraining conformal field theories with a higher spin symmetry, J. Phys. A 46 (2013) 214011 [arXiv:1112.1016] [InSPIRE].

[53] Y.S. Stanev, Correlation functions of conserved currents in four dimensional conformal field theory, Nucl. Phys. B 865 (2012) 200 [arXiv:1206.5639] [INSPIRE].

[54] A. Petkou, Conserved currents, consistency relations and operator product expansions in the conformally invariant $O(N)$ vector model, Annals Phys. 249 (1996) 180 [hep-th/9410093] [INSPIRE].

[55] N. Boulanger, D. Ponomarev, E.D. Skvortsov and M. Taronna, On the uniqueness of higher-spin symmetries in AdS and CFT, Int. J. Mod. Phys. A 28 (2013) 1350162 [arXiv: 1305.5180] [INSPIRE].

[56] A. Sagnotti and M. Taronna, String lessons for higher-spin interactions, Nucl. Phys. B 842 (2011) 299 [arXiv: 1006.5242] [inSPIRE].

[57] A. Fotopoulos and M. Tsulaia, On the tensionless limit of string theory, off-shell higher spin interaction vertices and BCFW recursion relations, JHEP 11 (2010) 086 [arXiv:1009.0727] [INSPIRE].

[58] M. Taronna, Higher-spin interactions: four-point functions and beyond, JHEP 04 (2012) 029 [arXiv: 1107.5843] [INSPIRE].

[59] P. Dempster and M. Tsulaia, On the structure of quartic vertices for massless higher spin fields on Minkowski background, Nucl. Phys. B 865 (2012) 353 [arXiv:1203.5597] [INSPIRE]. 